\title{
Rabi oscillations of pinned solitons in spin chains: A route to quantum computation and communication
}

\author{
S. Bertaina,,${ }^{1, *}$ C.-E. Dutoit,${ }^{1}$ J. Van Tol, ${ }^{2}$ M. Dressel,${ }^{3}$ B. Barbara, ${ }^{4}$ and A. Stepanov ${ }^{1}$ \\ ${ }^{1}$ Aix-Marseille Université, CNRS, IM2NP UMR7334, F-13397 Marseille Cedex 20, France \\ ${ }^{2}$ Physics Department and the National High Magnetic Field Laboratory, Florida State University, \\ 1800 East Paul Dirac Drive, Tallahassee, Florida 32310, USA \\ ${ }^{3}$ Physikalisches Institut, Universität Stuttgart, D-70550 Stuttgart, Germany \\ ${ }^{4}$ Université Grenoble Alpes and CNRS, Institut Néel, F-38042 Grenoble, France \\ (Received 16 January 2014; revised manuscript received 23 July 2014; published 20 August 2014)
}

\begin{abstract}
We provide evidence for the coherence and Rabi oscillations of spin solitons pinned by the local breaking of translational symmetry in isotropic Heisenberg chains (simple antiferromagnetic Néel or spin Peierls). We show that these correlated spin systems that are made of hundreds of coupled spins bear an overall spin $S=1 / 2$ and can be manipulated as a single spin. This is clearly contrary to known spin qubits which are paramagnetic centers, highly diluted to prevent decoherence. These results offer an alternative approach for spin qubits, paving the way for the implementation of a different type of quantum computer.
\end{abstract}

DOI: 10.1103/PhysRevB.90.060404

PACS number(s): 75.10.Pq, 03.67.-a, 76.30.-v

Most physical, chemical, or biological systems showing quantum oscillations are of relatively small size: for example, isolated nitrogen-vacancy $(\mathrm{NV})$ centers in diamond [1], $4 f$ or $3 d$ transition-metal ions (single spins, $0.1 \mathrm{~nm}$ ) [2,3], single molecule magnets [4] (15 spins, $1 \mathrm{~nm}$ ), or marine algae (5 $\mathrm{nm}$ wide proteins) [5]. Their environmental couplings are necessarily weak in order to reduce damping [6]. In magnetic systems, decoherence is usually dominated by spin-bath dipole-dipole interactions [7], and observations of quantum oscillations require qubit dilution. Here, we report an experimental realization of what we call soliton qubits. Contrary to existing qubits, each qubit is made of hundreds of strongly exchange-coupled spins sitting at the defects of strongly correlated spin chains. This takes advantage of one of the most remarkable properties of one-dimensional spin systems: their quite unconventional response to translational symmetry breaking which often consists in the formation of magnetic defects described as spin solitons (kinks or domain walls). In as-grown single crystals, these defects are generally associated with local inhomogeneities such as crystallographic defects or disorder, bond alternations, chain ends, etc. Theoretical predictions for Heisenberg spin 1/2 quantum spin chains show that each of these magnetic defects carry an overall spin $S=1 / 2$ which can serve as a qubit. The idea of a pinned soliton was successfully applied to explain the magnetic susceptibility [8], NMR [9], and some electron paramagnetic resonance (EPR) experiments [10] in spin chains, however, to date, little is known either experimentally or theoretically about the coherent dynamics of soliton.

In this Rapid Communication, we report on Rabi oscillations of pinned spin solitons in isotropic Heisenberg chains (simple antiferromagnetic Néel or spin Peierls). We demonstrate that these collective extended defects bear an overall spin $S=1 / 2$ and can be manipulated as single quantum spins. This clearly presents an alternative to known spin qubits which are paramagnetic centers in highly diluted

\footnotetext{
*sylvain.bertaina@im2np.fr
}

samples. These results offer an approach for spin qubits, paving the way for the implementation of a different type of quantum computer.

Evidence of Rabi oscillations in sol-qubits is obtained on single crystals of the so-called antiferromagnetic quantum spin chains (TMTTF) $2 X$, with $X=\mathrm{AsF}_{6}, \mathrm{PF}_{6}, \mathrm{SbF}_{6}$ (Fig. 1). This family of organic magnets, also called Fabre salts [11], was extensively studied during the last decades and shows an extremely rich phase diagram [12]. The systems with $X=\mathrm{AsF}_{6}$ and $\mathrm{PF}_{6}$ show a gapped dimerized spin-pair singlet ground state below their spin-Peierls transitions at $T_{\mathrm{SP}}=13$ and $19 \mathrm{~K}$, respectively, whereas the system with $X=\mathrm{SbF}_{6}$ exhibits a Néel antiferromagnetic phase below $T_{N}=7 \mathrm{~K}$.

The Hamiltonian of an $S=1 / 2$ Heisenberg chain can be written as

$$
H=J \sum_{i}\left[(1-\delta) S_{2 i-1} S_{2 i}+(1+\delta) S_{2 i} S_{2 i+1}\right],
$$

where $S_{i}$ are the $S=1 / 2$ spin operators, $J$ the exchange coupling, and $\delta$ the dimerization parameter. If $\delta=0$, (1) describes the uniform Heisenberg antiferromagnetic (AF) chain: The ground state is a gapless $S=1 / 2$ doublet. This is the case of the spin-chain system (TMTTF) $)_{2} \mathrm{SbF}_{6}$ at $T>T_{N}$. If $\delta>0$, (1) describes a spin-Peierls chain: The ground state is a gapped dimerized spin-pair singlet $(S=0)$ at temperatures below $T_{\mathrm{SP}}$. This is the case for the systems (TMTTF) $)_{2} \mathrm{PF}_{6}$ and (TMTTF) ${ }_{2} \mathrm{AsF}_{6}$ (if $T>T_{\mathrm{SP}}, \delta=0$ in these systems, too). The isotropic part of the exchange interaction of these three systems, $J \sim 400 \mathrm{~K}$, is relatively large whereas their intra- and interdimer contributions of (TMTTF) $)_{2} \mathrm{PF}_{6}$ and $(\mathrm{TMTTF})_{2} \mathrm{AsF}_{6}$ give a bond alternation (dimerization) parameter $\delta \sim 0.03$, leading to the singlet-triplet gap $\Delta=$ $35 \mathrm{~K}$ [14]. Such a value is more than enough to provide an excellent separation of the ground state at the Kelvin scale of temperatures, i.e., an extended collective singlet ground state in which two trapped soliton qubits can be strongly entangled.

The single crystals of (TMTTF $)_{2} X$ were grown by an electrochemical technique [15]. The crystals are needle shaped with typical dimensions $3 \times 0.5 \times 0.1 \mathrm{~mm}^{3}$. They crystallize 

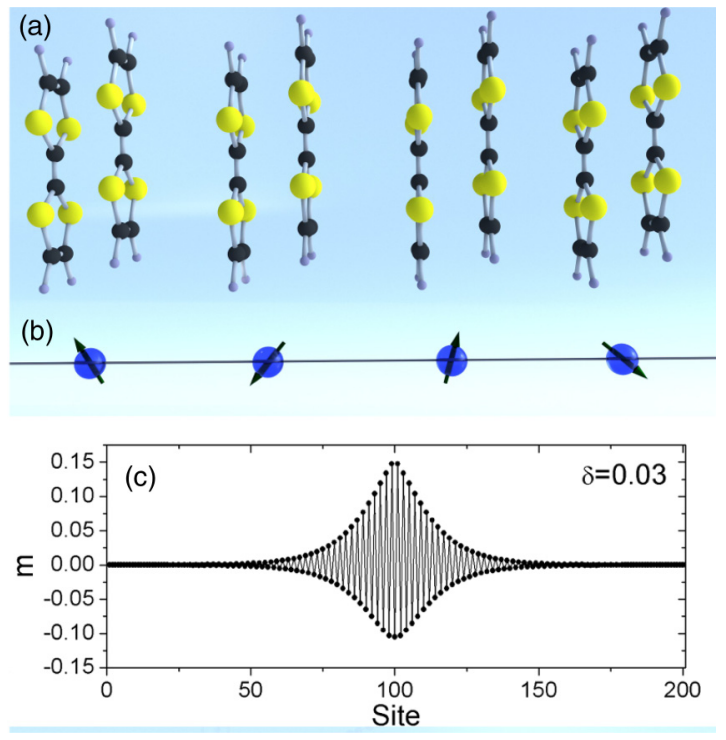

FIG. 1. (Color online) (a) Schematic representation of a (TMTTF) ${ }_{2} X$ chain. The TMTTF molecules are stacked in the $a$ axis direction, forming the chain. Each double molecule of TMTTF carries a spin $S=1 / 2$. The counteranion $X$ is not represented here. (b) Magnetic representation of the chain. The blue spheres are the double molecules of TMTTF carrying a spin (black arrow) and coupled along the $a$ axis by the exchange $J(1 \pm \delta)$. (c) Site magnetization profile induced by one bond defect placed in the middle of a dimerized chain in the $M z=1 / 2$ space computed by the density matrix renormalization group (DMRG) (see the method discussed in Ref. [13]).

in the triclinic $P \overline{1}$ space group. The magnetic principal axes $\left(b^{\prime}\right.$ and $\left.c^{*}\right)$ are different from the crystallographic axes and correspond to the extrema of the $g$ factor in the plane perpendicular to the $a$ axis. The static magnetic field can be applied in any direction in the $b^{\prime}-c^{*}$ plane. For each set of measurements a fresh sample was used.

Continuous wave $(\mathrm{cw})$ and pulsed electron paramagnetic resonance experiments were performed with the three systems using a conventional $X$-band Bruker spectrometer operating at about $9.6 \mathrm{GHz}$ between 3 and $300 \mathrm{~K}$ and enabling sample rotations. The crystals were glued on the sample holder with their $a$ axis oriented along the microwave field $\left(h_{\mathrm{mw}}\right)$ direction, which is the same as the sample-rotation direction (the static $H$ being applied in the basal $b^{\prime}-c^{*}$ plane with $\theta$ the angle between $H$ and $\left.c^{*}\right)$.

Above $30 \mathrm{~K}$ a single Lorentzian-shaped EPR line (main line) is observed, displaying an anisotropy of the $g$ factor, associated with different orientations of $H$. This anisotropy and the temperature dependence are typical of uniform quantum Heisenberg spin chains and were intensively studied in the past [16]. Below about $30 \mathrm{~K}$ a second EPR line, a very sharp one, appears in the three systems at the same magnetic field as the main line. The integrated intensity of this sharp signal (SS) is much smaller (by a factor of $10^{2}-10^{3}$ ) than the one of the main line, indicating its defect origin.

As an example, Fig. 2(a) gives a set of spectra obtained with $X=\mathrm{AsF}_{6}$ between 30 and $3 \mathrm{~K}$ with $H \| c^{*}$ showing how the very sharp signal progressively appears and becomes dominant
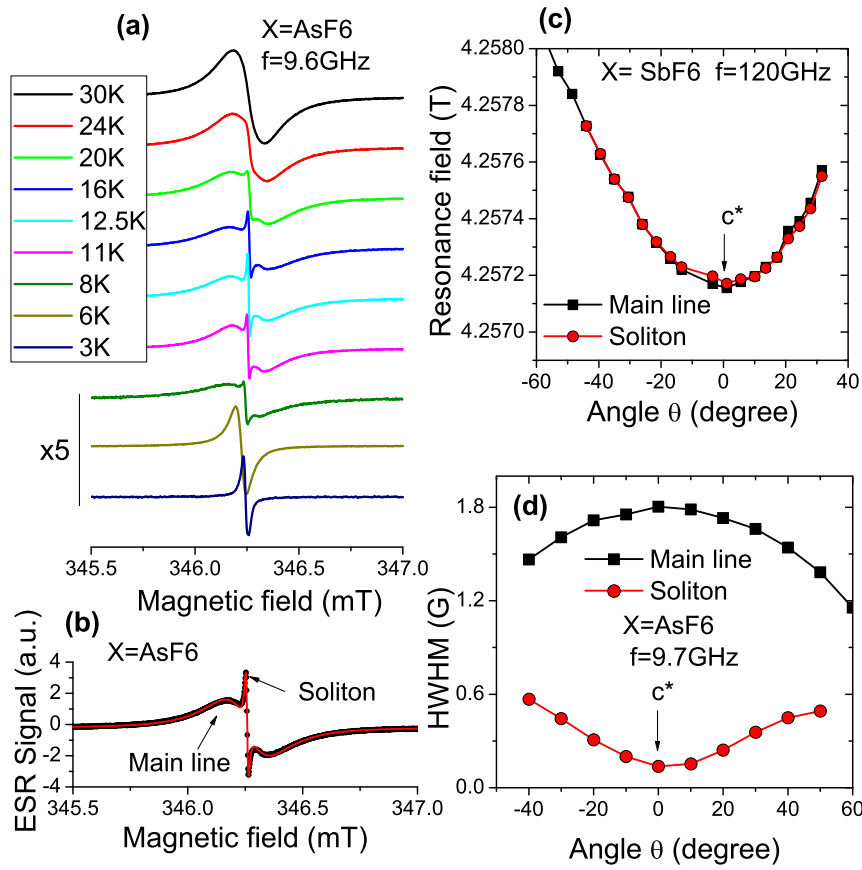

FIG. 2. (Color online) cw EPR, evidence of the soliton signal. (a) Set of cw EPR spectra of (TMTTF $)_{2} \mathrm{AsF}_{6}$ when the temperature decreases for $H \| c^{*}$. (b) EPR spectrum of (TMTTF) ${ }_{2} \mathrm{AsF}_{6}$ at $T=$ $12.5 \mathrm{~K}$. The dots are the experiments and the red line is a fit of 2 derivative of Lorentzian. (c) Angular dependence of the resonance fields of (TMTTF $)_{2} \mathrm{SbF}_{6}$ at $120 \mathrm{GHz}$. The black squares are the main lines, and the red circles are the soliton lines. (d) Angular dependence of the linewidth of (TMTTF) ${ }_{2} \mathrm{AsF}_{6}$ at $9.5 \mathrm{GHz}$ and $T=12.5 \mathrm{~K}$.

when the temperature decreases. Below $T_{\mathrm{SP}}=13 \mathrm{~K}$ the system starts to dimerize and enters into the spin-Peierls phase. The intensity of the main peak drops but that of the SS remains almost unchanged. The same behavior has been observed in the $\mathrm{PF}_{6}$ compound. In the $\mathrm{SbF}_{6}$ system, the linewidth of the main line diverges when $T$ decreases down to $T_{N}=$ $7 \mathrm{~K}$ whereas the SS remains almost unchanged, except below $T_{N}$, where it disappears [17]. This behavior is contrary to that of an isolated paramagnetic impurity, the intensity of which is strongly temperature dependent (Curie law), and is rather characteristic of an ensemble of correlated spins.

In the limit of the resolution of the $X$-band spectrometer, the measured resonance field and therefore the $g$ factor were identical for both the broad and sharp peaks and did not change significantly for different molecules. A two-Lorentzian fit shows that their linewidths differ by a factor of ten $[\sim 1 \mathrm{G}$ for the former and $\sim 0.1 \mathrm{G}$ for the latter, Fig. 2(b)]. In order to improve the resolution we measured the cw EPR in these systems at $120 \mathrm{GHz}$ in a homemade quasioptical cw EPR spectrometer [18] [the angular dependence of the magnetic field resonance of the $\mathrm{SbF}_{6}$ system is given in Fig. 2(c)]. The resonance fields of both lines remain identical for all the applied-field orientations, to an accuracy of better than $10^{-5}$.

Figure 2(d) gives the linewidth angular dependence of the two peaks observed. The broad/sharp linewidth ratio reaches its maximum of $\sim 10$ when the static field is applied along the $c^{*}$ axis. Surprisingly, whereas the resonant field of the two peaks is observed to be the same whatever the angle $\theta$, 
the angular dependences of their linewidths are opposite. The width of the broad line follows the well-known behavior of a uniform $S=1 / 2$ chain, proportional to $1+\cos ^{2} \theta$, with a maximum for $\theta=0^{\circ}$, whereas the one of the sharp line is minimum for $\theta=0^{\circ}$, as expected for a line issued from defectinduced correlated spins.

To sum up, while the SS has quite unusual temperature and linewidth angular dependencies, compared to an isolated impurity signal, one of the most important features which supports its spin soliton origin is the finding that the SS $g$ factor is precisely the same as the one of the main EPR line. This result is perfectly consistent with a physical picture which one can suggest to describe the defect formation in TMTTF charge transfer salts. As we have mentioned above, any translation symmetry breaking of the molecular structure in the direction of the chain, and crystallographic or electron charge inhomogeneities, will lead locally to an intrachain exchange modification which in turn will cause a spin soliton formation close to this defect. The observed EPR signal in this case will come not from an impurity center itself (a localized electron somewhere in TMTTF molecule) but from a collective precession of hundreds of nonperturbed spins of the chain which form a soliton. Quite naturally this precession is characterized by the same $g$ factor as the main EPR signal.

Before going further in our experimental investigation, let us explain in more detail the condition of the appearance and stability of solitons near the bond defects in spin chains. For that we have performed numerical DMRG studies of alternating spin chains in which the successive bonds are $J 1=J(1+\delta)$ and $J 2=J(1-\delta)$ with $J 1>J 2$. In Fig. 1(c) a typical distribution of the soliton magnetic moment is shown for a defect characterized by the succession of two strong bonds placed in the middle of the chain. The result, shown in Fig. 1, fully corresponds to a spin soliton, and after integration we find that it carries a value of $S=1 / 2$. These results are in full agreement with similar studies that have been previously published $[19,20]$ for comprehensive theoretical studies.

The pulsed EPR experiments were performed on $(\mathrm{TMTTF})_{2} \mathrm{AsF}_{6}$ and $(\mathrm{TMTTF})_{2} \mathrm{PF}_{6}$ single crystals with a microwave field $h_{\mathrm{mw}}$ varying between 0.1 and $1.5 \mathrm{mT}$. The coherent signal, resulting from the SS observed in cw experiments, was recorded by the free induction decay (FID) method. The spin-echo detection cannot be used here, in contrast with most other known systems, because of the absence of sizable inhomogeneous line broadening. In fact, the line broadening of $\sim 0.1 \mathrm{G}$ observed is essentially homogeneous (see the discussion below). Examples of Rabi oscillations obtained at $3 \mathrm{~K}$ for $X=\mathrm{PF}_{6}$ are given in Fig. 3. The oscillations are very well fitted by the exponentially damped sinusoidal function $\left\langle S_{x}(t)\right\rangle \propto \sin \left(\Omega_{R} t\right) \exp \left(-t / \tau_{R}\right)$, with $\Omega_{R}$ the Rabi pulsation and $\tau_{R}$ the Rabi damping characteristic time. The Rabi frequency increases linearly with $h_{\mathrm{mw}}$ with a slope $d\left(\Omega_{R} / 2 \pi\right) / d h_{\mathrm{mw}} \sim 28 \mathrm{MHz} / \mathrm{mT}$ close to the expected value for spins $S=1 / 2$ [Fig. 3(b)], thus providing additional evidence in favor of our model of a trapped soliton with $S=$ $1 / 2$. This figure also shows the microwave-field dependence of the Rabi damping $1 / \tau_{R}$, allowing one to evaluate the damping by the microwaves [2,4,21]. This "overdamping" that is associated with inhomogeneous line broadening due to the distributions of the transverse $g$ factor (itself resulting
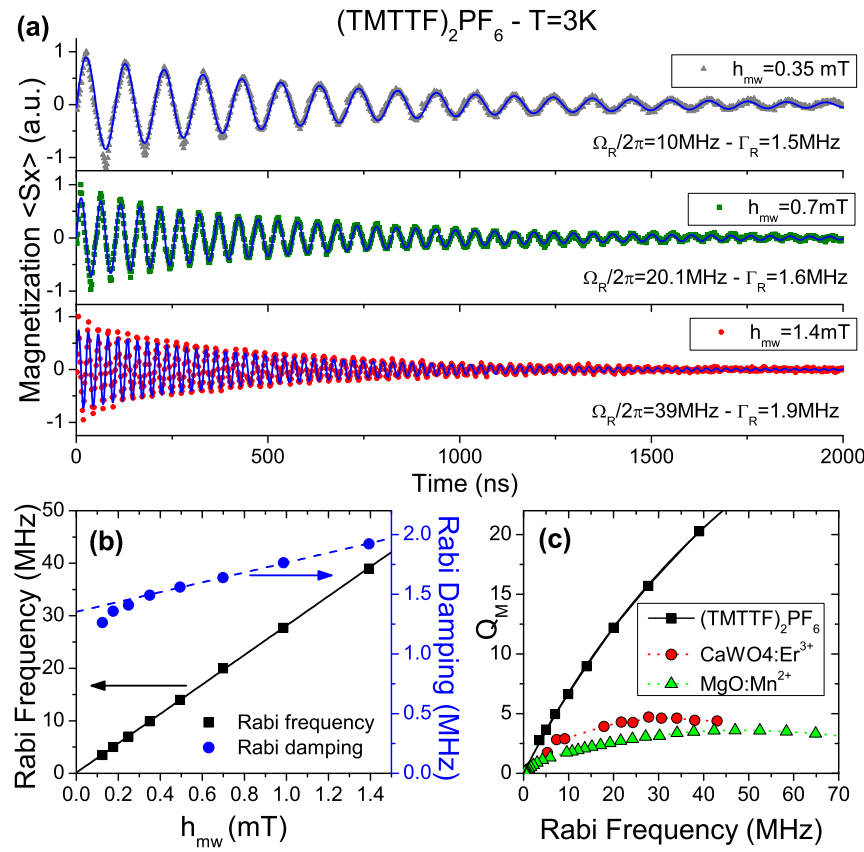

FIG. 3. (Color online) Rabi oscillations and coherence properties of a soliton qubit. (a) Series of Rabi oscillations of (TMTTF) ${ }_{2} \mathrm{PF}_{6}$ measured at $T=3 \mathrm{~K}$ and $f=9.7 \mathrm{GHz}$ using the FID method while increasing the microwave magnetic field $h_{\mathrm{mw}}$. Each point is an average of 1000 FID measurements. The blue lines are fits using $\sin \left(\Omega_{R}\right) \exp \left(t / \tau_{R}\right)$. (b) Rabi frequencies $\left(\Omega_{R} / 2 \pi\right)$ and damping $\left(1 / \tau_{R}\right)$ as functions of $h_{\mathrm{mw}}$. The black line represents the Rabi frequency of $\operatorname{spin} S=1 / 2$. The blue line is a fit using $\Gamma_{0}+\gamma h_{\mathrm{mw}}$, $\Gamma_{0}=1.35 \mathrm{MHz}$ (the zero microwave field coherence), and $\gamma=0.41$ $\mathrm{MHz} / \mathrm{mT}$ the effect of the microwave field on the Rabi damping. (c) Merit factor $Q_{M}$ of (TMTTF) $)_{2} \mathrm{PF}_{6}$ compared to diluted ion systems [22]. The black line is a simulation using the $\Gamma_{0}$ and $\gamma$ from (b).

from weak ligand-field distributions) or to the microwave-field amplitude (nonhomogeneous cavities) is generally unavoidable [22]. In the present case, the "overdamping" is particularly small due to the homogeneous character of the EPR line. The measured value of $d\left(1 / \tau_{R}\right) / d h_{\mathrm{mw}}=0.4 \mathrm{MHz} / \mathrm{mT}$ [Fig. 3(b)] is 10-50 times smaller than in ion-diluted systems [21,22]. In addition, contrary to most other systems, the figure of merit $Q_{M}=\Omega_{R} \tau_{R} / 2 \pi$ of sol-qubits in (TMTTF) ${ }_{2} \mathrm{PF}_{6}$ does not saturate when the Rabi frequency increases [Fig. 3(c)] and follows the expression $Q_{M}=\Omega_{R} /\left(8.5+0.015 \Omega_{R}\right)$. Our largest microwave field $h_{\mathrm{mw}}=1.5 \mathrm{mT}$ gives, at $3 \mathrm{~K}, Q_{M} \sim 23$, while a value of the order of 70 is expected for a larger field. Whereas $Q_{M}$ of $\mathrm{CaWO}_{4}: \mathrm{Er}^{3+}$ and $\mathrm{MgO}: \mathrm{Mn}^{2+}$ saturate at about 3, the $Q_{M}$ of sol-qubits is larger by an order of magnitude.

The fact that sol-qubits are almost insensitive to system parameters ( $g$ factors, inhomogeneous magnetic and microwave fields, etc.) is worthy of special consideration. Indeed, with other types of qubits these distributions lead to inhomogeneous distributions of Rabi frequencies (the qubits are not identical), giving destructive interferences and decoherence. This is a major roadblock for the implementation of a spin-based quantum computer. Magnetic dipole-dipole interactions are also inevitable and constitute a source of decoherence unless 
the qubits are very far from each other, preventing any kind of manipulation. With sol-qubits, the situation is just opposite: The strong spin-exchange interactions $(J=400 \mathrm{~K})$ eliminate decoherence through the well-known exchange narrowing mechanism, as this is shown in the $S=1$ Haldane spin chain [23] picture developed for interacting $S=1 / 2$ degrees of freedom. This explains why the coherence of sol-qubits is robust against microwaves even at high power. Finally, these long-living sol-qubits, even if they are distant, are easily coupled to each other and are controlled through an effective isotropic exchange interaction along the spin chain.

In conclusion, by observing long-living Rabi oscillations of sol-qubits in Heisenberg gapped spin-Peierls systems, we provide evidence for coherence in spin chains and, more particularly, in solitons trapped at exchange defects in spin chains. Due to an isotropic interqubit exchange interaction, the EPR lines observed are homogeneous and narrowed, eliminating the usual decoherence mechanisms such as the one associated with imperfectly identical qubits and dipolar interactions.
Following the idea of spin-qubit quantum computer [24], an increasing number of proposals were made during the last decades showing theoretically how spin chains may enable the implementation of a quantum computer by using them as quantum wires to connect distant qubit registers without resorting to optics [25-30]. We speculate that our sol-qubits might be ideal candidates for the realization of such a computer since they represent intrinsic spin registers which do not require the addition of any supplementary spin to the system, and since they perfectly match the communication channel, the spin chain.

We acknowledge the city of Marseille, Aix Marseille Université, NHMFL user program, and IR RENARD FR3443 for financial support. The NHMFL is supported by NSF Cooperative Agreement No. DMR-1157490, the U.S. Department of Energy, and by the State of Florida. We thank G. Gerbaud and PFM St. Charles for technical support. S.B. and B.B. thank I. Chiorescu and S. Miyashita for valuable discussions.
[1] L. Childress, M. V. Gurudev Dutt, J. M. Taylor, A. S. Zibrov, F. Jelezko, J. Wrachtrup, P. R. Hemmer, and M. D. Lukin, Science 314, 281 (2006).

[2] S. Bertaina, S. Gambarelli, A. Tkachuk, I. N. Kurkin, B. Z. Malkin, A. Stepanov, and B. Barbara, Nat. Nanotechnol. 2, 39 (2007).

[3] S. Bertaina, L. Chen, N. Groll, J. Van Tol, N. S. Dalal, and I. Chiorescu, Phys. Rev. Lett. 102, 050501 (2009).

[4] S. Bertaina, S. Gambarelli, T. Mitra, B. Tsukerblat, A. Müller, and B. Barbara, Nature (London) 453, 203 (2008).

[5] E. Collini, C. Y. Wong, K. E. Wilk, P. M. G. Curmi, P. Brumer, and G. D. Scholes, Nature (London) 463, 644 (2010).

[6] A. Leggett, S. Chakravarty, A. Dorsey, M. Fisher, A. Garg, and W. Zwerger, Rev. Mod. Phys. 59, 1 (1987).

[7] N. V. Prokof'ev and P. C. E. Stamp, Rep. Prog. Phys. 63, 669 (2000).

[8] J. Sirker, N. Laflorencie, S. Fujimoto, S. Eggert, and I. Affleck, Phys. Rev. Lett. 98, 137205 (2007).

[9] F. Alet and E. S. Sørensen, Phys. Rev. B 62, 14116 (2000).

[10] A. Zorko, D. Arčon, A. Lappas, J. Giapintzakis, C. Saylor, and L. C. Brunel, Phys. Rev. B 65, 144449 (2002).

[11] J. Moser, M. Gabay, P. Auban-Senzier, D. Jérome, K. Bechgaard, and J. Fabre, Eur. Phys. J. B 1, 39 (1998).

[12] D. Jérome, Science 252, 1509 (1991).

[13] B. Bauer, L. D. Carr, H. G. Evertz, A. Feiguin, J. Freire, S. Fuchs, L. Gamper, J. Gukelberger, E. Gull, S. Guertler, A. Hehn, R. Igarashi, S. V. Isakov, D. Koop, P. N. Ma, P. Mates, H. Matsuo, O. Parcollet, G. Pawowski, J. D. Picon, L. Pollet, E. Santos, V. W. Scarola, U. Schollwöck, C. Silva, B. Surer, S. Todo, S. Trebst, M. Troyer, M. L. Wall, P. Werner, and S. Wessel, J. Stat. Mech: Theory Exp. (2011) P05001.
[14] M. Dumm, B. Salameh, M. Abaker, L. K. Montgomery, and M. Dressel, J. Phys. IV 114, 57 (2004).

[15] S. Yasin, B. Salameh, E. Rose, M. Dumm, H.-a. Krug von Nidda, A. Loidl, M. Ozerov, G. Untereiner, L. Montgomery, and M. Dressel, Phys. Rev. B 85, 144428 (2012).

[16] B. Salameh, S. Yasin, M. Dumm, G. Untereiner, L. Montgomery, and M. Dressel, Phys. Rev. B 83, 205126 (2011).

[17] C. Coulon and R. Clérac, Chem. Rev. 104, 5655 (2004).

[18] J. van Tol, L.-C. Brunel, and R. J. Wylde, Rev. Sci. Instrum. 76, 074101 (2005).

[19] M. Nishino, H. Onishi, K. Yamaguchi, and S. Miyashita, Phys. Rev. B 62, 9463 (2000).

[20] M. Nishino, H. Onishi, P. Roos, K. Yamaguchi, and S. Miyashita, Phys. Rev. B 61, 4033 (2000).

[21] J. H. Shim, S. Bertaina, S. Gambarelli, T. Mitra, A. Müller, E. I. Baibekov, B. Z. Malkin, B. Tsukerblat, and B. Barbara, Phys. Rev. Lett. 109, 050401 (2012).

[22] H. De Raedt, B. Barbara, S. Miyashita, K. Michielsen, S. Bertaina, and S. Gambarelli, Phys. Rev. B 85, 014408 (2012).

[23] P. P. Mitra, B. I. Halperin, and I. Affleck, Phys. Rev. B 45, 5299 (1992).

[24] D. Loss and D. P. DiVincenzo, Phys. Rev. A 57, 120 (1998).

[25] W. K. Wootters, Phys. Rev. Lett. 80, 2245 (1998).

[26] M. C. Arnesen, S. Bose, and V. Vedral, Phys. Rev. Lett. 87, 017901 (2001).

[27] G. L. Kamta and A. F. Starace, Phys. Rev. Lett. 88, 107901 (2002).

[28] S. Bose, Phys. Rev. Lett. 91, 207901 (2003).

[29] L. Campos Venuti, C. Degli Esposti Boschi, and M. Roncaglia, Phys. Rev. Lett. 96, 247206 (2006).

[30] L. Amico, A. Osterloh, and V. Vedral, Rev. Mod. Phys. 80, 517 (2008). 